\title{
Mungi-Castañeda $\mathbf{S}^{1}$ Perona-Miguel de Priego $\mathbf{G}^{2}$
}

${ }^{1}$ CD, Esp. Odontopediatría, Maestrando en Odontopediatría Universidad Científica del Sur Lima-Perú.

2 CD, Esp. Odontología Pediátrica, Mg. Odontopediatría Coordinador Maestría Universidad Científica del Sur Lima-Perú.

\section{ESTRATEGIA DE SALUD BUCAL PARA NIÑOS CON DISCAPACIDAD VISUAL}

\section{RESUMEN}

El propósito de este trabajo es presentar una propuesta de la Guía de Salud Bucal para niños con discapacidad visual ya que es una preocupación del Programa de Maestría en Odontopediatría de la UCSUR la inclusión de personas en este caso con discapacidades visuales, al acceso de información sobre la importancia de la salud bucal y no habiendo encontrado en nuestro medio este material educativo, es que esta Guía fue desarrollada dentro de uno de los proyectos de innovación y producción académica; esto es un inicio, que con seguridad servirá como base para desarrollar futuros trabajos.

PALABRAS CLAVE: Salud bucal, discapacidad visual, niños; Braille.

\section{ORAL HEALTH STRATEGY FOR VISUALLY IMPAIRED CHILDREN}

\section{ABSTRACT}

The aim of this paper is to present an approach of an Oral Health Guide for children with visual disabilities. One of the concern of the Master Program in Pediatric Dentistry of UCSUR is the inclusion of people with disabilities. Nowadays this kind of educative material is missing. This guide was developed into one of the innovation projects and academic production of the Pediatric Dentistry Master Program; this is a start, which certainly will serve as basis for developing future works.

KEY WORDS: Orak health, visually impaired, children; Braille.

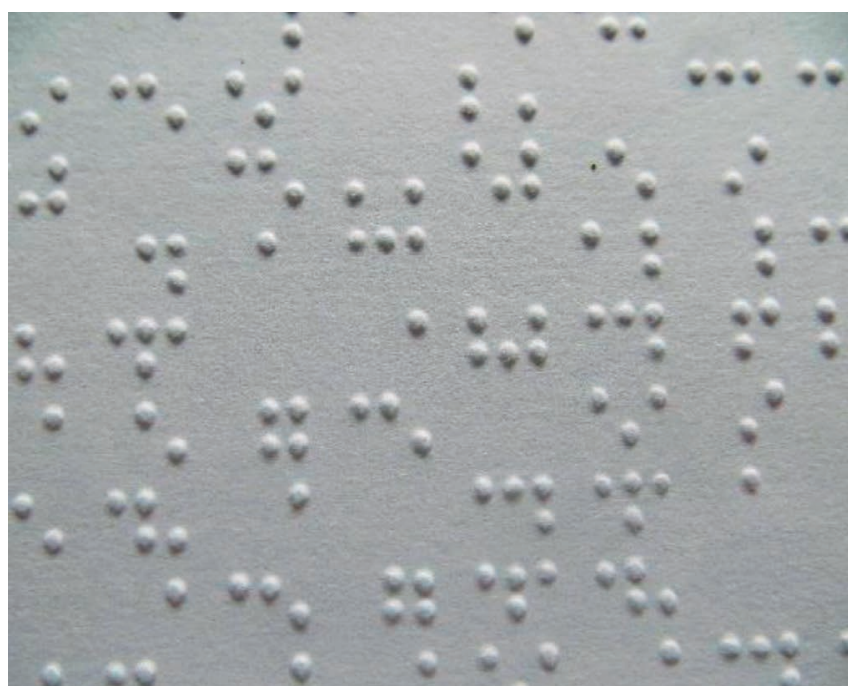




\section{INTRODUCCIÓN}

La Organización Mundial de la Salud en la 56 Asamblea Mundial de la Salud en su punto 14.17 del orden del día 28 de mayo de 2003, reconoce que 45 millones de personas en el mundo de hoy son ciegas y que otros 135 millones de personas son discapacitadas visuales; reconoce que el $90 \%$ de las personas ciegas y discapacitadas visuales del mundo entero viven en los países más pobres del mundo; advierte el significativo impacto económico de esta situación tanto en las comunidades como en los países. ${ }^{1}$

La Organización Mundial de la Salud (OMS) determinó una clasificación de la agudeza visual, estableciendo cuatro grupos diferentes según la visión del mejor ojo con la corrección óptica disponible en el momento del examen. Estos grupos son: ceguera, limitación visual severa (LMS), limitación visual (LV) y normalidad. ${ }^{2,3}$

Como ceguera se identifica la agudeza visual menor a 20/400 (0,05 o 3/60). La limitación visual severa comprende el grupo de personas que logran una agudeza visual de menos de 20/200 $(0,1)$ hasta 20/400. En el grupo de limitación visual, los individuos alcanzan agudezas visuales entre 20/60 (0,33) hasta 20/200 y finalmente, los del grupo identificado como de normalidad logran una agudeza visual de 20/60 o más. ${ }^{3,4}$

Un estudio estadístico de la propia OMS, demostró que en el año 2010 en la población mundial 50 millones de habitantes presentaban ceguera reversible e irreversible, de cuya cifra 1,5 millones eran menores de 16 años. ${ }^{4}$ Esta prevalencia varía entre $0,25 \%$ y $1,4 \%$ de acuerdo al nivel de desarrollo de la zona contemplada. Si sumamos a las cifras anteriores el número de personas con limitación visual severa, tendríamos que hablar de 180 millones de habitantes afectados, de los cuales el $90 \%$ se encuentran en países en vía de desarrollo. ${ }^{6}$ De igual manera, se determinaron las causas de ceguera y de LVS más importantes, entre las cuales la catarata fue la más frecuente (50\%), seguida del glaucoma (16\%), el tracoma
(12\%), la retinopatía diabética (8\%), la ceguera infantil (3\%), (retinopatía de la prematuridad y deficiencia de vitamina A, entre otros) y los defectos refractivos no corregidos $(8 \%){ }^{3}$

El mantenimiento de la higiene bucal es una de las tareas difíciles, junto con otras tareas para discapacitados visuales. Cuando se compara con personas sin discapacidad visual las personas con discapacidad visual tendrán más pobre la higiene oral y alto índice de caries. Los estudios realizados por Venugopal ${ }^{5}$ en India, Vignehsa et $a 1 .{ }^{6}$ en Singapure, y Reddyt et al. ${ }^{7}$ en el sur de la India atribuyen que se ha encontrado una mala salud bucal en niños especiales. Desafortunadamente, los métodos convencionales de enseñar el mantenimiento de la higiene oral como el uso de recursos visuales, agentes reveladores de placa no ayudan a los discapacitados visuales que dependen del tacto y la sensación para aprender. ${ }^{8}$

La salud bucodental de las personas con discapacidad visual puede estar en desventaja, ya que no están en condiciones de detectar y reconocer la enfermedad oral precoz y pueden ser incapaces de tomar medidas inmediatas. De ahí que la instrucción adecuada y el cuidado apropiado de los dientes y los tejidos orales son esenciales. Los discapacitados visuales dependen mucho más del sonido, el habla y el tacto, para orientarlos a una situación. De ahí que la educación para la salud bucal debe ser modificada para adaptarse a su discapacidad. ${ }^{9}$

Mejoras importantes en la salud bucal se han producido en muchos países desarrollados durante los últimos 30 años. Sin embargo las enfermedades orales son todavía muy extendidas en los países en desarrollo y su impacto en la sociedad y el individuo son significativas. Los costos del tratamiento son altos, y si las causas de las enfermedades son conocidas; se pueden prevenir en gran medida. ${ }^{10}$

Se han sugerido una serie de factores como jugando un papel en la motivación de los pacientes con discapacidad visual para llevar una bue- 
na higiene oral. El más importante entre estos factores son el reconocimiento de un paciente de la enfermedad y el conocimiento de diversas medidas preventivas. La situación se vuelve más compleja cuando se trata de discapacidad visual; ya que tienen problemas de salud oral similares a los observados en la población general. ${ }^{11}$ Es necesario hacer hincapié en la importancia del cuidado oral para estos individuos. ${ }^{12}$ Ellos deberían ser guiados en la eliminación y / o reducción de la biopelícula dental y la promoción de la salud. El conocimiento también debe ser impartido con respecto a la funcionalidad y la conservación de los elementos dentales y la estética, aunque podrían no tener un alta prioridad las personas con discapacidades visuales que motivan impedimentos para tener una buena higiene bucal, debido a que las técnicas típicas utilizadas en fisioterapia oral no serán usadas eficazmente. ${ }^{13}$ Las técnicas deben ser diseñadas conforme a las necesidades de los pacientes, se recomienda guías de salud escritas en el lenguaje Braille. ${ }^{14}$

Estudios sobre la educación para la salud bucal para personas con discapacidad visual son muy escasas. Por lo tanto el presente artículo tiene como objetivo presentar una Guía de Salud Bucal en lenguaje Braille para educar a personas con discapacidad visual respecto al cepillado de dientes, alimentos cariogénicos y causas de la caries. ${ }^{15,16,17,18}$

\section{PROPUESTA DE GUIA DE SALUD BUCAL EN BRAILLE (SM-GP)}

Los autores no habiendo encontrado una Guía de Salud Bucal para niños con discapacidad visual es que realizaron una búsqueda en diversos medios, luego realizaron visitas y entrevistas a personas y niños con discapacidad visual y a educadores, maestros especializados para conocer el interés, lo que llevó a desarrollar el presente proyecto.

Este se desarrolló con ayuda de educadores, personas y niños con discapacidad para poder llegar a interpretar la simbología Braille, también en buscar una imprenta especializada para editar y publicar. (Fig. 1-5).

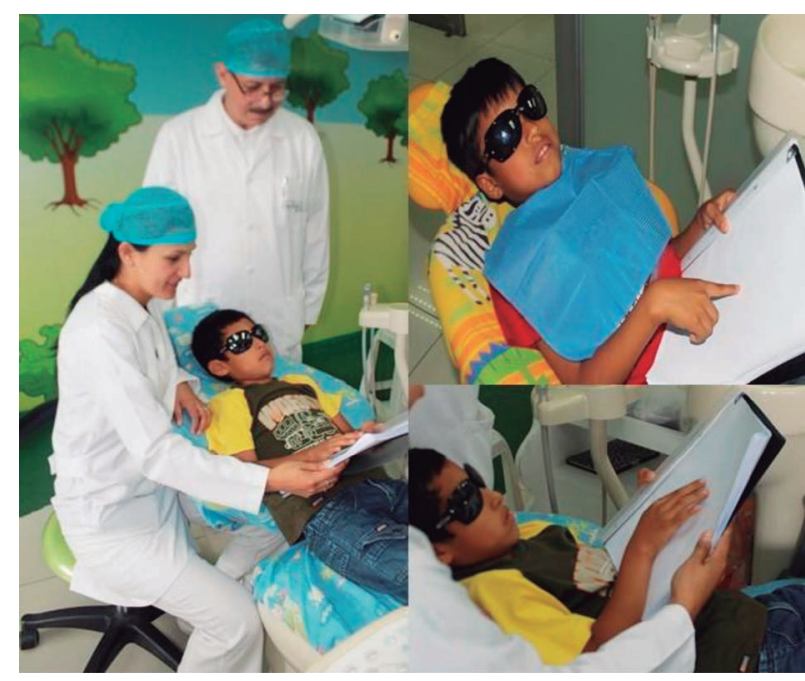

GUÍA DE SALUD BUCAL EN BRAILLE

Figura 1. Guía de Salud Bucal en Braille
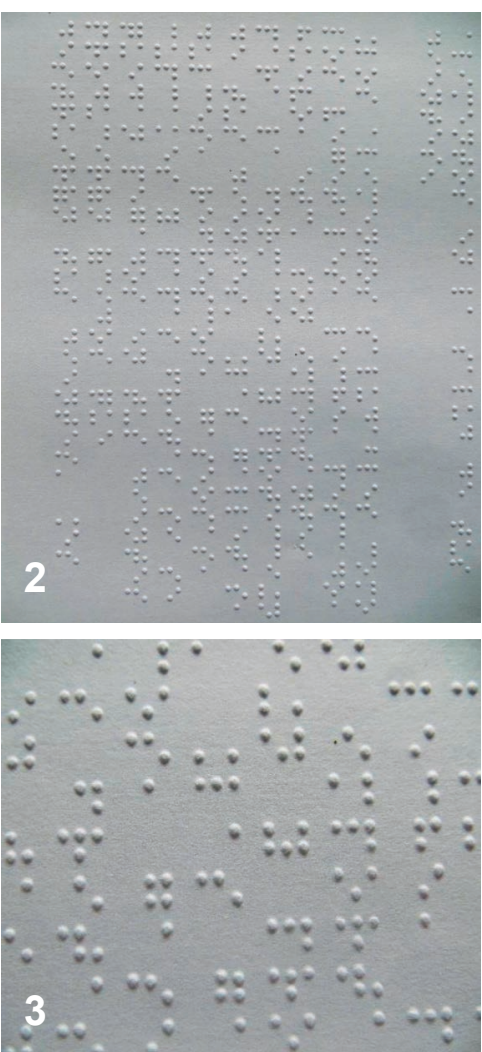

Figura 2 y 3. Imágenes de texto en Braille de la Guía de Salud Bucal 

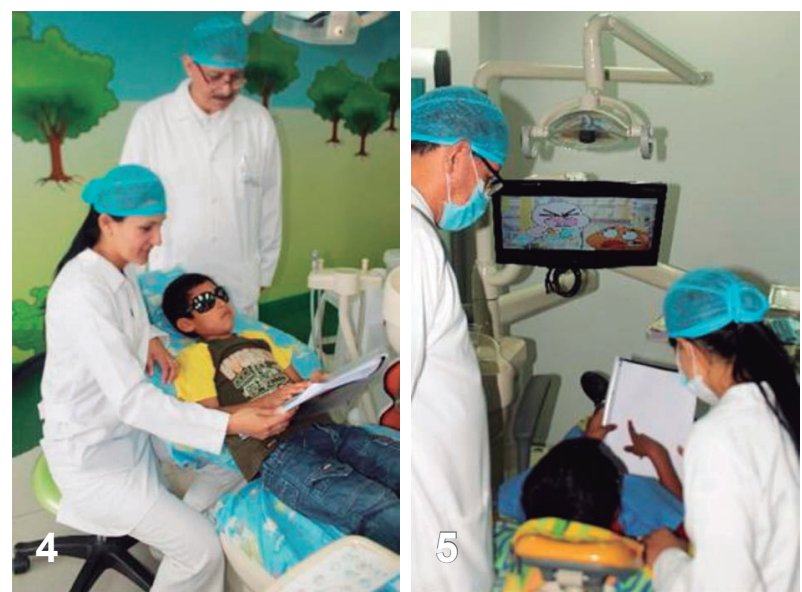

Figura 4 y 5. Autores enseñando la utilización de la Guía a niños con discapacidad visual

Luego de las entrevistas se confeccionó un borrador y después se realizaron múltiples sesiones educativas de diferentes niños con discapacidades visuales encontrando una gran motivación y capacidad de entendimiento sobre el contenido de la Guía, luego se dió el paso final de la edición e impresión.

Esta Guía ha sido entregada a la Escuela de Estomatología de la UCSUR para que pueda seguir desarrollándose y beneficiar en forma inclusiva a las personas con estas discapacidades.

\section{RECOMENDACIONES}

Se debe realizar y desarrollar otras propuestas de Salud Bucal inclusivas para personas con discapacidades y difundir la presente.

\section{REFERENCIAS BIBLIOGRÁFICAS}

1. World Health Organization. Disabilities. Available from: http://www.who.int/topics/disabilities/en/. [Last accessed on 2016 Feb 22].

2. World Health Organization. World report on disability. Available from: http://www.who.int/ disabilities/world_report/2011/report/en/index. html. [Last accessed on 2016 Feb 10].

3. World Health Organization. Visual impairment and blindness. Available from: http://www.who. int/mediacentre/factsheets/fs282/en/index.html. [Last accessed on 2012 Feb 10].

4. World Health Organization. Vision 2020 the right to sight. Global initiative for the elimination of avoidable blindness: Action plan, 2006-2011. Available from: http://www.who.int/ blindness/2020_report.pdf. [Last accessed on 2016 Feb 8].

5. Venugopal K., Eshwar K. Reddy E. A comparison of oral hygiene status and dental caries experience among institutionalized visually impaired and hearing impaired children of age between 7 and 17 years in central India. Journal of Indian Society of Pedodontics and Preventive Dentistry 2013; 31: 141-45.

6. Vignehsa H, Soh G, Lo GL, Chellappah NK: Dental health of disabled children in Singapore. Aust Dent J 1991, 36(2):151-156.

7. Reddy K, Sharma A: Prevalence of oral health status in visually impaired children. J Indian Soc Pedod Prev Dent 2011, 29(1):25-27.

8. Bedi R, Champion J, Horn R. Attitudes of the dental team to the provision of care for people with learning disabilities. Spec Care Dentist 2001; 21:147-52.

9. Deborah RS, Moffett, Lieberman, Dummer GM. Perceived Competence of Children with Visual Impairments. J Vis Impair Blind 2005; 99:15-25.

10.Hebbal M, Ankola A.V. Development of a new technique (ATP) for training visually impaired children in oral hygiene maintenance. European archives of Paediatric Dentistry 2012; 13 (5): 244-47.

11.Mahantesha T, Nara A, Kumari PR, Halemani PK, Buddiga V, Sarpangala M. A comparative evaluation of oral hygiene using Braille and audio instructions among institutionalized visually impaired children aged between 6 years and 20 years: A 3-monthfollow-up study. J Int Soc Prevent Communit Dent 2015; 5: S129-32.

12. Nandini NS: New insights into improving the oral health of visually impaired children. J Indian Soc Pedod Prev Dent 2003, 21(4):142-143.

13. Shetty V, Hegde AM, Bhandary S, Rai K: Oral health status of the visually impaired children-a south Indian study. J Clin Pediatr Dent 2010, 34(3):213-216.

14. Titiyal JS, Pal N, Murthy GV, Gupta SK, Tandon $\mathrm{R}$, Vajpayee RB, et al. Causes and temporal 
trends of blindness and severe visual impairment in children in schools for the blind in North India. Br J Ophthalmol 2003; 87: 941-5.

15. Yalcinkaya SE, Atalay T. Improvement of oral health knowledge in a group of visually impaired students. Oral Health Prev Dent 2006; 4: 243-253.

16. Shih YH, Chang CHS. Knowledge of dental health and oral hygiene practices of Taiwanese visually impaired and sighted students. J Visual Imp and Blindness 2004; 98: 1-15.
17.Pascolini D, Mariotti S, Pokharel G, Pararajasegaram R, Etya'ale D, Negrel AD, et al. 2002 global update of available data on visual impairment: A compilation of population based prevalence studies. Ophthalmic Epidemiol 2004; 11: 67-115.

18. Rao D, Amitha H, Munshi AK. Oral hygiene status of disabled children and adolescents attending special schools of South Canara, India. Hong Kong Dent J 2005; 2:107-13.

Correspondencia: Sabina Mungi Castañeda

Dirección:saby1000@hotmail.com
Recibido: 10-02-2016

Aceptado: 20-04-2016 